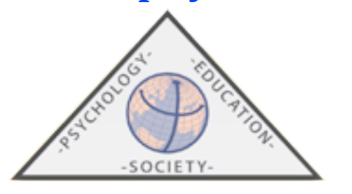

\title{
Caminos que se Consolidan en el Desarrollo Profesional Docente. ¿Están Presentes en España?
}

\author{
Elena MARTÍN
}

\author{
Universidad Autónoma de Madrid (España) \\ (Recibido el 24 de mayo de 2015; Aceptado el 2 de agosto de 2015)
}

\begin{abstract}
RESUMEN: El artículo tiene un doble objetivo. Por una parte, presentar un análisis de los principales rasgos que caracterizan los modelos y programas de Desarrollo Profesional Docente (DPD) considerados de mayor calidad hoy día. Este análisis se apoya en los artículos presentados en el monográfico, pero recoge también otros trabajos que complementan dicha perspectiva. Se constata que los ejes del cambio se articulan en torno al desarrollo a lo largo toda la carrera, la reflexión en comunidades de práctica que toman la escuela como unidad de referencia, y donde la voz de los alumnos va ganando presencia. Se destaca asimismo la necesidad de dar coherencia a las políticas de profesorado en el marco de las políticas globales de mejora de la calidad. El segundo objetivo consiste en valorar la situación del DPD en el sistema educativo español, tomando como criterio los ejes de análisis planteados. La comparación pone de manifiesto notables limitaciones, tanto desde el punto de vista de las actividades de formación del profesorado como del modelo global de desarrollo profesional docente.
\end{abstract}

Palabras clave: desarrollo profesional docente, colaboración entre profesores, comunidades de aprendizaje, práctica reflexiva

\section{Pathways that Converge in Teacher Professional Development. Are They Present in Spain?}

\begin{abstract}
The aim of this article is twofold. First, we present an analysis of the main features of the teacher professional development (PD) models and programs currently considered of highest quality. This analysis is supported by the articles presented in the present monograph, and it also considers other studies that complement these perspectives. We show that axes of teacher change deal with career-long development, reflection in school-based communities of practice, and focus on students' voices. In addition, we highlight the need for coherence between teacher policies and more global policies aimed at enhancing the quality of education. The second objective is to assess the situation of teacher PD within the Spanish education system, using the identified axes of teacher change as an
\end{abstract}


analytic framework. The comparison reveals important limitations from the points of view of both the specific PD activities offered to teachers and underlying teacher PD model.

Keywords: teacher professional development, collaboration among teachers, learning communities, reflective practice

Correspondencia: Elena Martín Ortega. Catedrática de Psicología de la Educación. Facultad de Psicología. Universidad Autónoma de Madrid. 28049 Madrid. Phone: (+34) 914975176. E-mail: elena.martin@uam.es

\section{Cómo citar este artículo}

Martín, E. (2015). Caminos se consolidan en el desarrollo profesional docente. ¿Están presentes en España?, Psicología, Sociedad y Educación, 7(3), 443-458.

\section{Introducción}

Hoy en día, tanto entre los académicos como entre los responsables de la política educativa, existe un amplio consenso acerca de la crucial importancia del profesorado en la mejora de la calidad de la enseñanza (European Commission/EACEA/Eurydice, 2013; MacBeath, 2012; Mourshed, Chijioke, \& Barber, 2010; UNESCO-OREALC, 2014), aunque por desgracia, este acuerdo no siempre se traduce en el impulso de políticas coherentes con dicho convencimiento. No resulta difícil identificar una serie de supuestos teóricos acerca del aprendizaje y cambio del profesorado sobre los que existe un amplio consenso, que a su vez coinciden con la realidad de determinados sistemas educativos que destacan por su compromiso con la innovación educativa (Avalos, 2011; Borko, 2004; Vaillant \& Marcelo, 2015; Stigler \& Hiebert, 2009; Villegas-Reimers, 2003).

En los cinco artículos que componen este monográfico, expertos en el campo del Desarrollo Profesional Docente (DPD) analizan las experiencias de sus respectivos países (Australia, Finlandia, Hong Kong, Estados Unidos y Singapur). En los textos se aprecian importantes coincidencias en las prácticas que se vienen impulsando desde las administraciones educativas de sus países. En la primera parte de este artículo, se exponen los que consideramos ejes esenciales del avance en los modelos de DPD, que se reconocen en gran medida en las cinco experiencias anteriores. En la segunda, se analiza la realidad del sistema educativo español a la luz de los supuestos enunciados.

\section{De la actualización al desarrollo profesional}

El primero de los cambios se refiere al propio concepto de DPD. La concepción de un proceso centrado en la actualización de conocimientos ha dado paso a un enfoque en el que se trata de ofrecer al docente las condiciones para desarrollarse en todas las variadas y complejas dimensiones de su quehacer profesional. Un cambio que implica reestructurar sus concepciones, valores, actitudes y por supuesto prácticas. Un proceso dinámico e inacabado, que requiere tiempo y que debe estar presente a lo 
largo toda la trayectoria profesional. En último término, una exigencia de repensar qué significa en este momento ser profesor.

La naturaleza global, permanente y sustantiva de esta transformación se refleja en el análisis de los cinco artículos anteriores, pero es quizás la expresión que retoman Ling y Mackenzie (2015) la que mejor lo transmite. Estos autores aluden a la propuesta de Barnett (2012) que califica la profundidad del cambio de "ontological turn". Sin querer caer en afirmaciones grandilocuentes, es innegable que el desarrollo profesional docente se ve afectado por los profundos cambios que conlleva la sociedad de la información (Castells, 2000). En ella, aprender a lo largo de la vida es una necesidad esencial y el reto de la escuela es por tanto intentar que todos los alumnos y alumnas adquieran la competencia de aprender a aprender antes de finalizar la escolarización. La incertidumbre es la característica más definitoria de las sociedades actuales y es preciso que los nuevos ciudadanos afronten el futuro con flexibilidad intelectual, y sabiendo controlar la ansiedad emocional que provocan las situaciones en gran medida impredecibles. Los espacios informales ganan peso en el aprendizaje; la escuela no es ya el único lugar donde se construye conocimiento, lo que obliga a repensar su papel y a resituar su posición entre los distintos contextos educativos (Coll, 2013; Ito et al. 2013). Enseñar es ahora una tarea distinta y ser docente requiere redefinir la función de la educación escolar y de quienes deben ayudar a los estudiantes a construir conocimiento.

El profundo cambio que se aprecia en la caracterización de la figura del docente y en las formas de apoyar su desarrollo no solo se explica por las transformaciones sociales sino también por las que se han producido en la forma de entender el aprendizaje y la enseñanza. Los enfoques cognitivos se han enriquecido con la perspectiva sociocultural, como muestra Avalos (2011) en su ilustrativa revisión. La influencia de la cultura en las prácticas sociales educativas, la mediación entendida como ayuda a la construcción activa del aprendiz o las tecnologías como artefactos semióticos esenciales para el aprendizaje son algunos de los constructos teóricos que ilustran esta evolución (Sawyer, 2008; Wells, 1999). Ser profesor tiene otro significado desde una concepción sociocultural. Remite a un perfil profesional mucho más complejo. Retomando el enfoque de Scardamalia y Bereiter (2006), los docentes serían expertos capaces de resolver problemas abiertos en vez de afrontar los cambios con rutinas aprendidas previamente (Niemi, 2015).

El modelo de "Teacher Professional Growth" que Clark y Hollingsworth (2002) propusieron hace ya más de una década, al que hacen referencia varios de los autores del monográfico, refleja bien esta complejidad. Por una parte, el docente debe cambiar en distintos elementos de su persona y su tarea profesional. Por otra, el modelo hace énfasis en la influencia de las condiciones del contexto social y de la propia escuela en este cambio. Promoverlo implica tener en cuenta todos estos factores, pero interconectados entre sí. Como plantean Ling y Mackenzie (2015), a partir de Opfer y Pedder (2011), el aprendizaje del profesor no puede entenderse como un acontecimiento aislado sino como un sistema con distintos niveles de construcción $\mathrm{y}$, por tanto, también de intervención. Un sistema dinámico que 
evoluciona a lo largo del tiempo, lo que conlleva planificar y apoyar el desarrollo del profesor durante las dos-tres décadas que dura su carrera profesional.

\section{Reflexión y autorregulación: claves del desarrollo profesional}

La complejidad de la tarea docente requiere un profesional autónomo que analiza y comprende las demandas que se derivan de la realidad en la que se desenvuelve, planifica intencionalmente su actuación y la revisa de forma periódica y sistemática, modificándola a partir de los resultados obtenidos y de los cambios sociales que inciden en la función de la escuela. Enseñar desde este enfoque supone tener muy desarrolladas la capacidad de reflexión y de autorregulación. Cuando hace tres décadas Schön $(1983,1987)$ atacaba la racionalidad técnica que convertía al docente en mero ejecutor de lo que otros decían, su posición era todavía minoritaria. Hoy en día la concepción del docente como un profesional reflexivo es ampliamente compartida en el mundo académico, aunque esté lejos de haberse generalizado en la práctica de las escuelas.

Este enfoque tiene importantes consecuencias para la planificación del DPD. La primera es la concepción de aprendizaje en la que se basa, en concreto en lo que se refiere a las relaciones entre cognición y acción. El DPD es un proceso que tiene su origen en la reflexión sobre la práctica. No se produce tan solo a partir del acceso a nueva información. Supone por supuesto construir nuevo conocimiento, pero este se genera mediante la explicitación de las ideas y creencias que los profesores tienen y de su contraste y reelaboración a partir de la experiencia directa en el aula. Las actividades de formación docente deben implicar un análisis de la actividad del aula que permita ir dando nuevos significados a lo que allí sucede, cambiar algunos elementos de la enseñanza y revisar su efecto en el aprendizaje de los alumnos: un recursivo ciclo de reflexión sobre la práctica (Postholm, 2008; Pozo et al. 2010; Thorsen \& DeVore, 2013; Zeichner \& Liston; 1996). Los cursos o talleres de corta duración y desconectados de la actividad cotidiana del profesor pueden ser útiles para sensibilizar o para adquirir nueva información pero no permitirían, desde esta perspectiva, promover cambios que supongan un desarrollo profesional duradero.

En el apoyo a los procesos de reflexión y cambio, es necesario tener en cuenta los aspectos emocionales. Enfrentarse a la incertidumbre del cambio, en un panorama tan abierto y exigente como el que se ha descrito, genera ansiedad. No se trata por tanto de una resistencia únicamente cognitiva. Formar a un docente supone que el profesional más experto que le acompaña en el proceso anticipa estas dificultades y planifica la ayuda explicitando y normalizando estos sentimientos (Martín \& Cervi, 2006, Zembylas, 2010).

El anclaje en la práctica como origen del aprendizaje de los profesores conlleva a su vez atender a la importancia del contexto y del dominio de conocimiento. Los procesos de explicitación y reestructuración no son libres de contenido ni ajenos a la influencia del contexto, sino situados y específicos del ámbito disciplinar y didáctico (Avalos, 2011). Ello no significa, por supuesto, que no se produzca una generalización, pero esta no es sencilla ni inmediata. El conocimiento profesional abarca competencias comunes a distintas situaciones de enseñanza, además de las 
relativas a aprendizajes de ámbitos curriculares específicos, pero las primeras no se pueden construir en el vacío sino a partir de la reflexión sobre situaciones contextualizadas de la práctica docente (Clarke \& Hollingsworth, 2002; Nielsen, Barry, \& Staab, 2008; Wang, Kim, Lee, \& Kim, 2014).

El enfoque de la reflexión sobre la práctica resulta mucho más exigente para las administraciones educativas porque supone garantizar determinadas características en la cultura de los centros y en las condiciones laborales. Es preciso incluir en los horarios de los docentes tiempos para planificar y revisar las clases, que además deben poder compartirse con los colegas. No es realista pensar que todos los docentes van a dedicar espontáneamente horas a esta tarea tan ardua si además tienen que hacerlo fuera de su horario de trabajo. Los procesos de innovación, impulsados por las administraciones, que no tienen en cuenta la sobrecarga que pueden suponer para los docentes están condenados al fracaso. Dar clase no es la única tarea docente. Planificar, leer, pensar, re-pensar son actividades absolutamente necesarias para el DPD, que deben por tanto estar previstas en el horario laboral.

Los procesos de reflexión y explicitación se ven facilitados por la escritura (Klein, Boscolo, Gelati, \& Kirckpatrick, 2014; Scardamalia \& Bereiter, 2006). Es fundamental ir consolidando en los centros docentes la cultura de plasmar las decisiones en documentos, evitando desde luego convertir esta tarea en un proceso meramente burocrático. Escribir ayuda a pensar, permite compartir los proyectos y hace más fácil su revisión. Los equipos directivos y en general todos aquellos que ejercen liderazgo en la escuela deben promover la elaboración de documentos y su discusión y evaluación.

El papel de estas figuras resulta crucial en el diseño del desarrollo profesional de los profesores de su escuela (Harris, 2008; OCDE, 2009). Sin embargo, los docentes deben asumir su formación como una responsabilidad que ellos/ellas mismos deben planificar. Si bien es cierto que las necesidades de la institución deben estar presente en este plan, es esencial que el docente regule su propia trayectoria profesional. Se trata de un proyecto personal con el que es necesario sentirse comprometido. Como en otros muchos aspectos del desarrollo, se progresa en la medida en que se pasa de la heteroregulación a la autoregulación. Los líderes deben ayudar a elaborar el plan y a contrastar periódicamente sus logros. Este control y apoyo externo puede ayudar al docente, pero la iniciativa no puede estar solo fuera de él.

El impulso de la reflexión y la autorregulación choca no obstante con la tendencia que a menudo tienen las administraciones educativas a buscar que todos los profesores lleven a sus clases prácticas de enseñanza semejantes. Por desgracia, es bastante habitual que los responsables de las políticas educativas intenten generalizar la innovación deseada sin dar tiempo a que los profesores puedan hacerla suya. La tensión entre autonomía y rapidez y homogeneidad en el cambio es uno de los problemas del desarrollo profesional. El exponente más claro de este riesgo es el efecto perverso de determinadas formas de evaluación externa (Darling-Hammond, 2012). Como señala Avalos (2011), "Con el objetivo de conseguir mejores resultados en las pruebas de rendimiento, se les ofrecen "expertos de fuera" para 
enseñarles como producir resultados en el breve plazo que exigen los sistemas educativos" (p.18, la traducción es nuestra). La falta de autonomía se produce también por la presión que los docentes de algunos sistemas educativos viven por la exigencia de la sociedad sobre el nivel de rendimiento de los alumnos. En los artículos del monográfico que analizan la realidad de Hong Kong (Lam, 2015) y de Singapur (Bautista, Wong, \& Gopinathan, 2015) se llama la atención sobre este aspecto (Lam, 2015. Como contrapunto, Finlandia ha optado por un enfoque de autonomía y responsabilidad local que busca que los profesores puedan utilizar los métodos y formas de evaluación que mejor se adapten a sus alumnos y alumnas. La tarea de supervisión ha cambiado desde un enfoque de control a uno de apoyo y mentoría (Niemi, 2015).

\section{Del profesor al equipo docente}

Sabemos que los procesos de aprendizaje se ven favorecidos por la cooperación entre varias mentes, tanto por razones cognitivas como emocionales (Johnson, Johnson, \& Holubec, 2008, Salomon, 1993). Pensar juntos favorece la toma de conciencia sobre las ideas propias y promueve el perspectivismo y la argumentación. Ayuda a desarrollar la regulación del comportamiento tanto de uno mismo como de los demás y las actitudes de respeto y valoración de la diversidad.

Estos mecanismos que actúan en el caso de los estudiantes son igualmente válidos para el aprendizaje de los adultos, y entre ellos los profesores. Pero en este caso se suman otros argumentos que hacen especialmente valiosas estas estructuras de participación. La calidad de la enseñanza depende en gran medida de la coherencia entre las decisiones de los distintos docentes que ejercen influencia educativa sobre los mismos alumnos. La clave no está en la actuación aislada de cada profesor sino en la fuerza de las experiencias que se repiten a lo largo de la escolaridad porque responden a principios compartidos por el conjunto del equipo docente. No se trata de buscar la homogeneidad, pero sí la coherencia. El equipo docente pasa así a convertirse en la unidad de desarrollo profesional más potente. Quienes comparten un espacio de práctica educativa (docentes del mismo curso, de la misma materia, responsables de planes específicos...) pueden someter a análisis situaciones comunes, planificar cambios que comprometan a todos y apoyarse mutuamente durante el proceso. Estas políticas son las que se están impulsando en Australia, Finlandia, Hong Kong y Singapur.

Por ello, si bien las comunidades profesionales no tienen por qué limitarse a miembros de una misma institución escolar, los modelos de desarrollo profesional que toman el centro como unidad de intervención reúnen muchas de las ventajas que se vienen analizando. A las ya señaladas, se añadiría la mayor facilidad para conectar la formación docente con la mejora de la calidad de la enseñanza. Desde este enfoque es más sencillo identificar las necesidades de formación de los docentes en el marco de las necesidades de la escuela y hacer revertir las competencias adquiridas por estos en los planes de mejora del centro. Como señalan Darling-Hammond y McLaughlin (2003) "En diversos aspectos del trabajo cotidiano en las escuelas ya existen 
oportunidades para este tipo de aprendizaje y reflexión. Se puede decir que todo lo que sucede en una escuela presenta una oportunidad de desarrollo profesional" (p.20).

El papel de las figuras de liderazgo queda más claro y es más relevante desde este enfoque, tanto desde el punto de vista del apoyo que pueden prestar a los docentes como del seguimiento de sus planes. La figura de los mentores resulta fundamental desde este enfoque. Su presencia impulsa estos procesos de reflexión, focaliza los temas más relevantes, ofrece prácticas alternativas, asumiendo durante todo el proceso un liderazgo distribuido que caracteriza las experiencias de innovación (Hobson, Ashby, Malderez, \& Tomlinson, 2009).

En los últimos años, las experiencias de comunidades de práctica se han enriquecido con la incorporación de la perspectiva de los alumnos. Los estudios sobre "Lesson Study" muestran que, si bien han ido ganando presencia como vías de DPD, la investigación sobre sus efectos todavía no es del todo concluyente (Hart, Alston, \& Murata, 2011). Se trata de experiencias satisfactorias para los profesores, pero también de procesos costosos que requieren apoyo por parte de los equipos directivos de los centros (tiempo, recursos materiales y una figura que asesore a los docentes) (Lim, Lee, Saito, \& Hairon, 2011). En este mismo sentido se orientan las "comunidades epistémicas", basadas en el enfoque de Scardamalia y Bereiter de "knowledge building community school" (1994, 2006), que han mostrado ser una interesante vía de DPD (Wang, Kim, Wen, Lee, \& Kim, 2014). Ambas experiencias suponen líneas de avance muy interesantes que deberían por ello ser promocionadas por las administraciones.

En estas experiencias, a la fuerza de la reflexión sobre la acción se suma, por una parte, la potencia de la observación de otro docente y, por otra, el valor de la perspectiva de los estudiantes. Las comunidades de práctica ya suponen una revisión de la práctica entre pares, pero la relevancia de las sugerencias que otros colegas pueden plantear al observar directamente la clase y la utilidad de las sugerencias de mejora que pueden derivarse tienen el valor añadido de la actividad directa y contextualizada. La voz de los alumnos aporta una perspectiva que difícilmente pueden ofrecer otros docentes; no necesariamente más valiosa, pero desde luego complementaria. La presencia de la voz de los alumnos es un elemento clave de la educación inclusiva (Booth \& Ainscow, 2011; Messiou et al., en prensa). El enfoque de las "comunidades de aprendizaje" enriquece el contraste de perspectivas y conocimientos con la presencia de otros adultos de la comunidad (INCLUD-ED Consortium, 2011).

A pesar de la potencia que tiene el DPD situado en la escuela, las comunidades de práctica se articulan en otros casos en estructuras que reúnen a profesionales de distintas instituciones educativas. Los seminarios o grupos permanentes de trabajo, que comparten una misma área curricular o proyecto de enseñanza, los equipos que elaboran materiales didácticos, o los docentes que forman parte de proyectos de investigación de la universidad, son experiencias que promueven el DPD y pueden enriquecer la perspectiva interna de cada centro (Darling-Hamond \& McLaughing, 2003). Las redes de escuelas o entre instituciones de un abanico más amplio permiten también esta riqueza de intercambio de experiencias. El "Plan de Lecciones 
Colaborativo" (The Collaborative Lesson Planning), organizado por el Education Bureau de Hong Kong es un ejemplo de este tipo de programas. Entendemos que uno de los retos de las políticas de DPD es promover que este crecimiento del docente revierta finalmente en el conjunto de la escuela; encontrar el equilibrio entre la amplitud y variedad de formatos de aprendizaje y el papel privilegiado de los espacios de reflexión con los colegas con los que se comparte la práctica.

\section{Las políticas de profesorado en el marco de la mejora de la calidad}

La calidad de la formación en servicio siempre se ha visto afectada por el resto de las políticas de profesorado, pero esta dependencia se ve mucho más clara desde un enfoque de desarrollo profesional que entiende este concepto como una evolución a lo largo del proceso de construcción de la identidad y las competencias del docente. La forma de entender qué significa ser profesor y la importancia otorgada a la reflexión conjunta sobre la práctica deberían informar el curriculum de la formación inicial. La necesidad de una fase de inducción responde a estos mismos supuestos y es un ejemplo paradigmático de las borrosas fronteras entre formación inicial y permanente. Asimismo, las decisiones de evaluación del profesorado (función meramente formativa o con consecuencias para la carrera); el papel de los equipos directivos; los estándares de acreditación, repercuten directamente en el DPD (Darling-Hammond, 2012). Una clave del éxito en el diseño del DPD sería, por lo tanto, cuidar la coherencia en el diseño de estas políticas globales de profesorado, lo que implica hacer claramente explícito el modelo por el que se está optando (Villegas-Reimers, 2003).

Es importante tener en cuenta, no obstante, que esta coherencia debe ir más allá del ámbito del profesorado. Otras decisiones de estructura y funcionamiento del sistema educativo tienen también una clara incidencia sobre la viabilidad de poner en marcha un determinado modelo de DPD. El diseño del curriculum es sin duda una de ellas. ¿Qué concepción de aprendizaje se transmite? La enseñanza no puede organizarse al margen de lo que se quiere que los alumnos aprendan y de cómo se concibe el conocimiento y el aprendizaje. Existe una relación directa entre las competencias docentes y el curriculum escolar. Por estas mismas razones, es necesario revisar con sumo cuidado las opciones relativas a la evaluación externa del rendimiento de los alumnos, como se ha señalado más arriba.

Las decisiones que influyen en la cultura y organización de las escuelas resultan igualmente importantes. ¿Se promueve en la normativa y en las políticas un liderazgo distribuido y alejado del estilo burocrático (OCDE, 2009)? ¿Existen los tiempos y las estructuras para que los docentes puedan reunirse con otros colegas del centro? ¿Se tiene en cuenta en el reparto de responsabilidades la competencia de los docentes? ¿Se impulsan programas de mentoría? ¿Se tiene en cuenta la voz de los estudiantes? Estos indicadores, que caracterizan el grado en que una escuela tiene una cultura innovadora, dependen en parte de la gestión del propio centro, pero en último término responden a decisiones de política educativa de las administraciones que permiten o no las condiciones necesarias para una enseñanza de calidad. 
Ello no significa que deban limitarse al uso de sus propios recursos. Las universidades y los centros de investigación educativa son en muchos países parte muy activa en estos procesos, como se pone de manifiesto en los artículos del monográfico. En estos casos el apoyo al desarrollo profesional de los docentes se realiza en el marco de la colaboración entre centros escolares y universidad mediante su incorporación a equipos de investigación, lo que revierte además en el beneficio del conocimiento académico. Hay también numerosos ejemplos de la aportación de la iniciativa privada al DPD. Los titulares de redes de centros privados suelen contar con departamentos propios de formación. Los movimientos de renovación pedagógica desempeñan también un importante papel en esta oferta. Sin embargo, si la formación a lo largo de la carrera se entiende como un derecho y un deber de los docentes, es preciso que esté garantizado por los poderes públicos.

Finalmente, entendemos que también es responsabilidad de las administraciones comprobar el impacto de las políticas de DPD, aunque para ello necesiten la ayuda de los investigadores. Los datos con los que se cuenta hasta el momento resultan todavía ambiguos. Ante todo hay que reconocer la dificultad de concluir a partir de estudios que son muy diversos tanto en los objetivos, como en las metodologías utilizadas. De ahí que los resultados no puedan considerarse concluyentes, como señalan en sus artículos Bautista et al. (2015) y Ling y Mackenzie (2015). Algunos investigadores consideran que no se han encontrado datos que permitan afirmar que los programas estén teniendo impacto (e.g., DarlingHammond, 2010; Hoekstra \& Korthagen, 2011). Sin embargo, otras revisiones sí apuntan a que, cuando los programas de DPD reúnen las características señaladas (reflexión sobre la práctica; larga duración; en colaboración con otros colegas, en contextos de clase concretos), se aprecian cambios en las formas de enseñar de los profesores implicados (Avalos, 2011; Hagger, McIntyre, \& Wilkin, 2013; McKernan \& McKernan, 2013, Villegas-Reimers, 2003).

Todavía más difícil resulta comprobar que los programas de DPD mejoren los aprendizajes de los alumnos. Son escasos los estudios con los que se cuenta. Sin embargo, es necesario comprobar esta dimensión del impacto, centrándose en el aprendizaje de contenidos específicos (Bautista et al., 2015; Bautista, Cañadas, Brizuela, \& Schliemann, 2015; Kazemi \& Hubbard, 2008). Aunque la diversidad de los contextos escolares hace difícil la generalización de los resultados, conviene buscar en este ámbito educativo, como se ha hecho en otros, elementos comunes que permitan comprender mejor el impacto de la actividad docente. Los procesos de enseñanza y aprendizaje son interactivos (Coll, Onrubia, \& Mauri, 2008; Sánchez, García, Rosales, De Sixte, \& Castellano, 2008). El rendimiento de los alumnos y alumnas no puede explicarse por tanto únicamente por lo que el profesor hace, pero la capacidad de ajuste del docente a las características del alumno y del contenido es la clave de la enseñanza. El análisis de la relación entre la práctica docente en el aula y los aprendizajes alcanzados es una de las líneas de avance en la que la investigación educativa tiene mucho que aportar. 


\section{EI desarrollo profesional docente en España}

Si se analiza la situación del DPD en el sistema educativo español de acuerdo con los supuestos expuestos hasta aquí, la imagen no es positiva. En el año 1984, en el marco de la reforma experimental que daría lugar a la ley de educación que mayor incidencia ha tenido en España desde la transición democrática hasta el momento actual (LOGSE, 1990), se adoptó el modelo de los “Teacher's Center" ingleses. Esto supuso una descentralización en la formación de los profesores y un aumento muy importante de las plantillas de los asesores que trabajaban en la formación permanente. Se llevó a cabo una estrategia de formación en cascada: expertos que formaban a otros profesionales que a su vez organizaban los cursos y actividades formativas del conjunto de los docentes. Por otra parte, en el desarrollo normativo de la LOGSE se estableció una mejora salarial asociada a la formación que todavía hoy sigue vigente en la mayoría de las Comunidades Autónomas. Si el docente realiza 100 horas de formación en seis años recibe un aumento de sueldo en torno al 4,5\%.

Como señala Imbernón (2014), esta fase de apuesta clara por la formación fue asumida por los gobiernos autonómicos hasta completarse, antes del año 2000, el proceso de transferencia de las competencias educativas a las 17 comunidades. Con algunas pequeñas diferencias, el modelo se mantuvo en la inmensa mayoría de los casos. Sin embargo, en las tres últimas décadas, se ha producido un debilitamiento de las estructuras de formación. Tan sólo seis comunidades han mantenido la red de centros de profesores en el conjunto de su territorio. En el resto se han eliminado, dejando únicamente un centro de referencia para cada provincia o unidad administrativa análoga, con la correspondiente disminución de la oferta formativa. Los responsables suelen aducir que esta falta de apoyo se debe a la profunda crisis económica en la que se encuentra sumida la sociedad española. Sin embargo, a estas razones hay que añadir el ciclo claramente conservador que caracteriza la política española, y dentro de ella las decisiones educativas. Las instituciones privadas cuentan en la mayoría de los casos con recursos propios para la formación de su profesorado y el apoyo a la escuela pública ha dejado de ser una prioridad para los gobiernos conservadores, que en este momento ostentan la mayoría tanto en la administración del estado como en muchas Comunidades Autónomas.

Por otra parte, hay que destacar que, incluso en los momentos de mayor apoyo al DPD, el modelo respondía a un enfoque de formación continua basado fundamentalmente en la impartición de cursos de actualización de conocimientos. Las actividades de formación en las propias escuelas, o incluso los seminarios y grupos de trabajo son minoritarios, aunque se intentan impulsar desde algunas administraciones (Manzanares \& Galván Bovaira, 2012; OCDE, 2009). Las comunidades de aprendizaje profesionales que llevan a cabo actividades como las propuestas en este monográfico (e.g., lesson study; experiencias de investigaciónacción; co-tutorías) tienen escasa presencia en la realidad educativa española. Asimismo, los Movimientos de Renovación Pedagógica, asociaciones que desempeñaron un papel esencial en la innovación y el desarrollo profesional de los docentes, han experimentado un notable retroceso fundamentalmente por la falta de apoyo de las administraciones. 
Como suele suceder, esta situación poco estimulante no se limita a la formación permanente sino que caracteriza el conjunto de las políticas de profesorado. En el curso 2009-2010 los estudios universitarios que permiten dar clase en Educación Infantil y Primaria pasaron de diplomatura (3 años) a grado (4 años). Sin embargo, a la carrera de magisterio siguen sin acceder los estudiantes con mejores notas de la Educación Secundaria, a diferencia de lo que sucede en otros países como Finlandia o Singapur. También hasta ese momento para ser profesor de Secundaria bastaba con obtener un certificado de aptitud pedagógica unánimemente considerado una formación de muy baja calidad. Desde 2009 los graduados en las distintas disciplinas deben cursar un master de un año de duración que, si bien supone un claro avance, sigue sin dar una respuesta adecuada al complejo perfil competencial de un docente de esta etapa educativa (Manso \& Martín, 2013). Como señala Escudero (2009) en una interesante comparación entre el Marco General de Competencias Docentes elaborado por el Gobierno de Hong Kong en 2008 y el master de profesorado de España, se aprecia que este sigue estando demasiado centrado en las disciplinas.

En relación con el acceso a la profesión, la oposición que da entrada a la función pública continúa siendo una prueba demasiado memorística. Por otra parte, los expertos en educación vienen reclamando una etapa de inducción al incorporarse a la profesión (Marcelo, 2009). Sin embargo, esta no se ha establecido, pese a que en su momento se debatió la posibilidad de hacerlo siguiendo un modelo semejante al de los médicos noveles (MIR) (Marchesi \& Martín, 2014) y de que hay una parte importante de los docentes que lo apoya (Valle \& Manso, 2014).

Tampoco se ha conseguido un acuerdo entre las distintas administraciones, ni con los sindicatos, en torno a la carrera docente. No se cuenta con un sistema estatal ni autonómico de evaluación ni de acreditación del profesorado. En el sistema educativo español apenas existen experiencias de evaluación sistemática de la tarea docente, lo que impide tanto su uso formativo como la contribución a la carrera docente. La situación de España en este aspecto de las políticas de profesorado pone de manifiesto un claro estancamiento con respecto al panorama internacional (OCDE, 2009).

Los docentes ingresan a la profesión con un salario razonable-algo menor en los centros privados-, pero se jubilan 30 años después sin haber experimentado apenas mejoras en sus condiciones laborales (OCDE, 2010) y sin vías para su desarrollo profesional. La autonomía de los centros escolares es sumamente reducida en España y el papel de los equipos directivos en la política de personal es prácticamente inexistente (Pont, Nusche, \& Moorman, 2008). Por último, la universidad sigue demasiado desconectada de la formación permanente, salvo honrosas excepciones, y no se promueve la participación de profesores de enseñanzas anteriores a la universidad en proyectos de investigación.

Quizás todo ello contribuya, junto con otros factores, a una situación de malestar docente y a que los profesores y profesoras consideren que no se valora su labor como realmente se merecen. Son varios los estudios en los que se muestra una clara diferencia entre el prestigio que la sociedad otorga al profesorado y el que ellos 
se atribuyen a sí mismos (Fundación BBVA, 2009; Fundación Europea Sociedad y Educación, 2013, Marcelo, 2011; OCDE, 2009). El prestigio que los ciudadanos muestran en las encuestas está sensiblemente por encima del que los docentes creen tener y por debajo del que querrían obtener, y de hecho es muy semejante al de otras profesiones liberales. Como señala Fernández Enguita (2009; Fernandez Enguita \& Riviére Gómez, 2010), esta visión pesimista es una de las características de la profesión docente en España.

Este panorama, poco estimulante, no significa que no haya experiencias muy interesantes en las que se reconocen una vez más los elementos que se han venido analizando. Afortunadamente, existen valiosos ejemplos, que se han señalado en los párrafos anteriores, que ponen de manifiesto que hay docentes que han sido capaces de enfrentarse a las políticas "fósiles", como Darling-Hammond y McLaughlin (2003) las denominan. Es alentador comprobar que las innovaciones que se llevan a cabo en las escuelas pioneras del sistema educativo español responden a las líneas de mejora de la calidad de los proceso de enseñanza y aprendizaje en general y del quehacer docente en particular que se han analizado en la primera parte del artículo (INCLUDE-ED, 2011; Ministerio de Educación, 2011; Mourshed et al., 2010). ${ }^{i}$ La diferencia con otros países, entre los que se encuentran los cinco cuya realidad se ha expuesto en los artículos del monográfico, es que en España estos casos son todavía poco frecuentes y no constituyen por tanto la tendencia general.

La situación del sistema educativo español pone claramente de manifiesto la dimensión cultural y sistémica del cambio que supone pasar de un modelo de actualización de conocimientos a uno de desarrollo profesional. Las ideas que vertebran este avance se conocen desde hace tiempo y se aceptan desde el punto de vista teórico (véase el monográfico de Revista de Educación sobre la tarea de enseñar; véase AA.VV., 2006). También en España se defiende desde la academia que el objetivo es que el docente pueda crecer como profesional a través de la reflexión conjunta sobre la práctica. Sin embargo, este modelo no se ha traducido de una manera clara ni generalizada en políticas educativas y no ha llegado por tanto a la mayoría de las escuelas. El cambio del que venimos hablando no depende de un profesor aislado, exige una cultura al menos del conjunto de la escuela y, en último término, del sistema escolar. En los países donde se avanza en esta línea, el cambio también ha sido lento. Las ideas siempre se han anticipado a las prácticas. Empezaron a aplicarse por vanguardias minoritarias, como suele suceder, pero en un momento determinado se asumieron por los responsables educativos que las tradujeron en condiciones para el cambio. El apoyo firme y sostenido en el tiempo por parte de las administraciones no basta para la transformación cultural que este modelo de DPD exige, pero sin él, no creemos posible el avance en este ámbito esencial de la calidad de la enseñanza.

La evidencia sólidamente fundamentada en este monográfico debería ser considerada por los responsables del Ministerio de Educación español y de las administraciones educativas autonómicas. Las políticas de profesorado tendrían que convertirse en una prioridad en el presupuesto dedicado a educación. El aumento de la inversión es imprescindible para todas las medidas que se han apuntado (menos 
horas de clase para poder participar en reuniones de equipo docentes; reducción horaria de los mentores; niveles de carrera docente; oferta de calidad de actividades de formación; colaboración con proyectos de investigación de la universidad). El incremento del presupuesto es un requisito ineludible, pero también lo es la aprobación de un marco normativo que regule el estatuto docente y la formación de los profesores noveles.

\section{Referencias}

AA.VV. (2006). La tarea de enseñar: atraer, formar, retener y desarrollar buen profesorado. Revista de Educación. Monográfico, 340.

Avalos, B. (2011). Teacher professional development in Teaching and Teacher Education over ten years. Teaching and Teacher Education, 27(1), 10-20.

Barnett, R. (2012). Learning for an unknown future, Higher Education Research \& Development, 31(1), 65-77.

Bautista, A. Wong, J., \& Gopinathan, S. (2015). Teacher professional development in Singapore: Depicting the landscape. Psicología, Sociedad y Educación, 7(3), 311-326.

Bautista, A., Cañadas, M. C., Brizuela, M. B., \& Schliemann, A. D. (2015). Examining how teachers use graphs to teach mathematics in a professional development program. Journal of Education and Training Studies, 3(2), 91106. doi: 10.11114 jets.v3i2.676

Booth, T. \& Ainscow, M. (2011). Index for inclusion: developing learning and participation in schools ( $3^{\text {rd }}$ edition). Bristol: Centre for Estudies in Inclusive Education (CSIE).

Borko, H. (2004). Professional development and teacher learning: Mapping the terrain. Educational Researcher, 33(8), 3-15. http://dx.doi.org/10.3102/0013189X033008003

Castells, M. (2000). La era de la información. Vol. 1. La sociedad red. Madrid. Alianza.

Clarke, D., \& Hollingsworth, H. (2002). Elaborating a model of teacher professional growth. Teaching and Teacher Education, 18, 947-967.

Coll, C. (2013). El currículo escolar en el marco de la nueva ecología del aprendizaje. Aula, 219, 31-36.

Coll, C., Onrubia, J. \& Mauri, T. (2008). Ayudar a aprender en contextos educativos: el ejercicio de la influencia educativa y el análisis de la enseñanza. Revista de Educación, 346, 33-70.

Darling-Hammond, L. (2010). Teacher Education and the American Future. Journal of Teacher Education, 61(1-2), 35-47. http://dx.doi.org/10.1177/0022487109348024

Darling-Hammond, L. (2012). Evaluating and supporting effective teaching: Developing a $\begin{array}{llll}\text { systemic approach. Pensamiento Educativo, } 49(2), & 1-20\end{array}$ doi:10.7764/PEL.49.2.2012.1

Darling-Hammond, L. \& McLaughlin, M.W. (2003). El desarrollo profesional de los maestros. Nuevas estrategias y políticas de apoyo. México, D.F.: Secretaría de Educación Pública.

Escudero, J. M. (2009). La formación del profesorado de Educación Secundaria: contenidos y aprendizajes docentes. Revista de Educación, 350, 79-103. 
European Commission/EACEA/Eurydice (2013). Key Data on Teachers and School Leaders in Europe. 2013 Edition. Eurydice Report. Luxembourg: Publications Office of the European Union.

Fernández Enguita, M. (2006). Pero... ¿qué querrán? El estatus del profesorado y el tópico del reconocimiento. Cuadernos de Pedagogía, 353, 80-85.

Fernández Enguita, M. \& Rivière Gómez, J. (Eds.) (2010). Barómetro del Profesorado. Escuela, 3.869, 3 de junio de 2010 (suplemento especial).

Fundación BBVA (2009). Participación política y social y confianza en grupos profesionales e instituciones. Recuperado el 17 de enero de 2015 en http://www.fbbva.es/TLFU/tlfu/esp/investigacion/fichainves/index.jsp?codigo =369

Fundación Europea Sociedad y Educación (Eds.) (2014). El prestigio de la profesión docente en España: percepción y realidad. Madrid: Fundación Europea Sociedad y Educación. Recuperado el 28 de febrero de 2014 en http://www.sociedadyeducacion.org/publicaciones/coleccion-propia/

Hagger, H., McIntyre, D., \& Wilkin, M. (Eds.) (2013). Mentoring: perspectives on schoolbased teacher education. London: Routledge.

Harris, A. (2008). Distributed leadership in schools: Developing the leaders of tomorrow. London: Routledge \& Falmer Press.

Hart, L. C., Alston, A., \& Murate, A. (Eds.) (2011). Lesson study research and practice in mathematics education: Learning together. Dordrecht, The Netherlands: Springer.

Hobson, A. J., Ashby, P., Malderez, A., \& Tomlinson, P. D. (2009). Mentoring beginning teachers: what we know and what we don't. Teaching and Teacher Education, 25(1), 207-216.

Hoekstra, A., \& Korthagen, F. (2011). Teacher learning in a context of educational change: Informal learning versus systematically supported learning. Journal of Teacher Education, 62(1), 76-92. http://dx.doi.org/10.1177/0022487110382917 http://www.fbbva.es/TLFU/tlfu/esp/investigacion/fichainves/index.jsp?codigo=369

INCLUDE-ED Consortium (2011). Actuaciones de éxito en las escuelas europeas. Madrid: Ministerio de Educación. Recuperado el 87 de diciembre de 2014 en http://www.nesetweb.eu/sites/default/files/actuaciones-de-exito-en-las-escuelaseuropeas.pdf

Imbernon, F. (2014). La formación permanente 40 años después: de la ilusión a los recortes. Cuadernos de Pedagogía, 451, 56-59.

Ito, M., Gutiérrez, K., Livingstone, S., Penuel, B., Rhodes, J., Salen, K., Schor, J., SeftonGreen, J. \& Watkins, S. C. (2013). Connected Learning: An Agenda for Research and Design. Irvine, CA: Digital Media and Learning Research Hub.

Johnson, D. W., Johnson, R. \& Holubec, E. (2008). Cooperation in the classroom. Edina, $\mathrm{MN}$ : Interaction Book Company.

Kazemi, E., \& Hubbard, A. (2008). New directions for the design and study of Professional Development. Attending to the coevolution of teachers' participation across contexts. Journal of Teacher Education, 59(5), 428-441.

Klein, P., Boscolo, C., Gelati, P., \& Kirckpatrick, L. (Eds.) (2014). Writing as a Learning Activity. Leiden, Netherlands: Koninklijke Brill.

Lam, B. H. (2015). Teacher Professional Development in Hong Kong Compared to Anglosphere: the Role of Confucian Philosophy. Psicología, Sociedad y Educación, 7(3), 295-310. 
Lim, C., Lee, C., Saito, E., \& Hairon, S. (2011). Taking stock of Lesson Study as a platform for teacher development in Singapore. Asia-Pacific Journal of Teacher Education, 39(4), 353-365. doi: 10.1080/1359866X.2011.614683

Ling, L. M. \& Mackenzie, N. M. (2015). An Australian perspective on teacher professional development in supercomplex times. Psicología, Sociedad y Educación, 7(3), 264-278.

MacBeath, J. (2012). Future of teaching profession. Cambridge: University of Cambridge and Educational International Research Institute.

Manso, J. \& Martín, E. (2014). Valoración del Máster de formación de profesorado de Educación Secundaria: Estudio de casos en dos universidades. Revista de Educación, 364, 145-169.

Manzanares, A. \& Galván-Bovaira, A. (2012). La formación permanente del profesorado de educación infantil y primaria a través de los centros de profesores. Un modelo de evaluación. Revista de Educación, 359, 431-455 DOI: 10-4438/1988-592X-RE-2010$359-101$.

Marcelo, C. (2009). Profesorado principiante e inserción profesional a la enseñanza. Barcelona: Octaedro.

Marcelo, C. (2011). La profesión docente en momentos de cambios. ¿Qué nos dicen los estudios internacionales? CEE Participación Educativa, 16, 49-68.

Marchesi, A. \& Martín, E. (2014). Calidad de la enseñanza en tiempos de crisis. Madrid: Alianza.

Martín, E. y Cervi. J. (2006). Modelos de formación docente para el cambio de concepciones en los profesores. En Nuevas formas de pensar el aprendizaje y la enseñanza. Concepciones de profesores y alumnos (pp. 420-434). Barcelona: Graó.

McKernan, J., \& McKernan, J. (2013). Curriculum action research: A handbook of methods and resources for the reflective practitioner (2nd Ed.). London: Routledge.

Messiou, K., Ainscow, M., Echeita, G., Goldrick, S., Hope, M., Paes, I., Sandoval, M., Simon, C. \& Vitorino, T. (in press). Learning from differences: a strategy for teacher development in respect to student diversity. School Effectiveness and School Improvement. doi:10.1080/09243453.2014.966726.

Ministerio de Educación-IFIIE (2011). Estudio sobre la innovación educativa en España. Madrid: Publicaciones del Ministerio de Educación.

Mourshed, M., Chijioke, Ch., \& Barber, M. (2010). How the world's most improved school systems keep getting better. Londres: McKinsey \& Company. Recuperado el 23 de enero del 2012 de http://mckinseyonsociety.com/how-the-worlds-most-improvedschool-systems-keep-getting-better/

Niemi, H. (2015). Teacher professional development in Finland: Towards a more holistic approach. Psicología, Sociedad y Educación, 7(3), 279-294.

OCDE (2009). Creating Effective Teaching and Learning Environments: First results from TALIS. París OCDE. Recuperado el 19 de febrero de 2013 en http://www.oecd.org/edu/school/43023606.pdf

OCDE (2010). Education at a Glance. 2010. París: OCDE. Recuperado el 16 de marzo de 20014 en http://www.oecd.org/edu/eag2010

Opfer, V. \& Pedder, D. (2011). Conceptualizing teacher professional learning. Review of Educational Research, 81(3), 376-407.

Pont, B., Nusche, D., \& Moorman, H. (2008). Improving school leadership. Vol. 1: Practice and policy. Paris: OCDE. 
Postholm, M. B. (2008). Teachers developing practice: Reflection as key activity. Teaching and Teacher Education, 24(7), 1717-1728.

Pozo, J. I., Martín, E., Pérez-Echeverría, M. P., Scheuer, N., Mateos, M. \& de La Cruz, M. (2010). Ni contigo ni sin ti... Las relaciones entre cognición y acción en la práctica educativa. Infancia y Aprendizaje, 33(2), 179-184. doi:10.1016/j.tate.2008.02.024

Salomon, G. (1993). No distribution without individuals" cognition: A dynamic interactional view. In Salomon, G. (Ed.), Distributed Cognitions (pp. 111-138). New York: Cambridge University Press.

Sánchez, E., García, J. R., Rosales, J, De Sixte, R \& Castellano, N. (2008). Elementos para analizar la interacción entre estudiantes y profesores: ¿qué ocurre cuando se consideran diferentes dimensiones y diferentes unidades de análisis? Revista de Educación, 346, 105-136.

Sawyer, R. K. (2008). Optimizing learning implications of learning sciences research. In CERI/OECD, Learning in the 21st Century: Research, Innovation and Policy. Paris: OECD Publications.

Scardamalia, M. \& Bereiter, C. (1994). Computer support for knowledge-building communities. The Journal of the Learning Sciences, 3(3), 265-283

Scardamalia, M. \& Bereiter, C. (2006): Knowledge building: Theory, pedagogy and technology. En R. K. Sawyer (Ed.), The Cambridge handbook of the learning sciences (pp. 97-115). New York: Cambridge University Press.

Schön, D. A. (1983). The Reflective Practitioner: How professionals think in action. London: Temple Smith.

Schön, D. A. (1987). Educating the Reflective Practitioner. San Francisco: Jossey-Bass.

Stigler, J. W., \& Hiebert, J. (2009). The teaching gap: Best ideas from the world's teachers for improving education in the classroom. New York, NY: Free Press.

Thorsen, C. A., \& DeVore, S. (2013). Analyzing reflection on / for action : A new approach. Reflective Practice: International and Multidisciplinary Perspectives, 14(1), 88-103. doi:10.1080/14623943.2012.732948

UNESCO-OREALC (2014). Temas críticos para formular nuevas políticas docentes en América Latina y el Caribe: el debate actual. Santiago de Chile: Unesco-Orealc.

Vaillant, D. \& Marcelo, C. (2015). El ABC y D de la formación docente. Madrid: Narcea.

Valle, J. \& Manso, J. (2014). La voz del profesorado: Acceso a la profesión docente e inserción en el puesto de trabajo. Consejo General de los Ilustres Colegios Oficiales de Doctores y Licenciados en Filosofía y Letras y en Ciencias. Recuperado el 7 de noviembre del 2014 en http://www.consejogeneralcdl.es/

Villegas-Reimers, E. (2003). Teacher professional development: an international review of the literature. Paris: International Institute for Educational Planning.

Wang, X., Kim, B. Lee, J \& Kim, M. S. (2014). Encouraging and being encouraged: Development of an epistemic community and teacher professional growth in a Singapore classroom. Teaching and Teacher Education, 44, 12-24.

Wells, G. (1999). Dialogic Inquiry. Cambridge, R.U: The Press Syndicate of the University of Cambridge.

Zeichner, K. \& Liston, D. (1996). Reflective Teaching: an introduction. Mahwah, New Jersey: Lawrence Erlbaum Associates.

Zembylas, M. (2010). Teacher emotions in the context of educational reform. In A. Hargreaves, A. Lieberman, M. Fullan \& D. Hopkins (Eds.), Second International Handbook of educational change (pp. 221-236). London: Springer. 
${ }^{\mathrm{i}}$ Las experiencias recogidas en las dos revistas nacionales de mayor presencia entre los docentes, Cuadernos de Pedagogía y Aula de Innovación, dan también buena cuenta de estas valiosas innovaciones. 